\title{
Staphylococcus aureus from atopic dermatitis skin alters cytokine production triggered by monocyte-derived Langerhans cell
}

Kazumasa Iwamoto ${ }^{1}$, Masaya Moriwaki ${ }^{1}$, Yoshie Niitsu1,2, Masachika Saino1, Junzo Hisatsune ${ }^{2}$, Motoyuki Sugai², Michihiro Hide ${ }^{1}$

1) Department of Dermatology, 2) Department of Bacteriology,

Graduate school of Biomedical and Health Sciences, Hiroshima University, Japan

\section{Background / Question addressed}

Patients with atopic dermatitis (AD) have frequent bacterial infections and $A D$ skin harbors an increased level of Staphylococcus aureus ( $S$ aureus). However, skin immune responses via activated Langerhans cell (LC) by different strains of $\mathrm{S}$. aureus, especially clinically isolated strains from AD skin, have not been studied.

The aim of this study is to explore whether $S$. aureus isolated from AD skin is able to skew $T$ cell responses via LC as compared to a control strain of $S$. aureus and $S$. epidermidis

\section{Material \& Method - 1}

CD14pos monocytes and CD4pos $\mathrm{T}$ cells were 1. Prepare MoLC. sorted from peripheral blood mononuclear cells of healthy controls $(\mathbf{H C})$ and patients with $\mathbf{A D}$. Monocyte-derived Langerhans cells (MoLC) were generated by culturing monocytes with GM-CFS, IL- 4 and TGF- $\beta$ for 7 days. MoLC were cultured with or without $S$. aureus (control and AD strains), S. epidermidis and Pam3CSK4 for $24 \mathrm{~h}$, then washed and subsequently sorted by CD1a ${ }^{\text {pos }}$ by FACSAria TM. Sorted CD1a ${ }^{\text {pos }}$ MoLC were co-cultured with autologous CD4pos $T$ cells, which were isolated and kept frozen until Mixed lymphocyte reaction. After co-culture, T cell functions were
analyzed. This study was approved by the local ethics committee of Hiroshima University and informed consent was obtained from all participants.

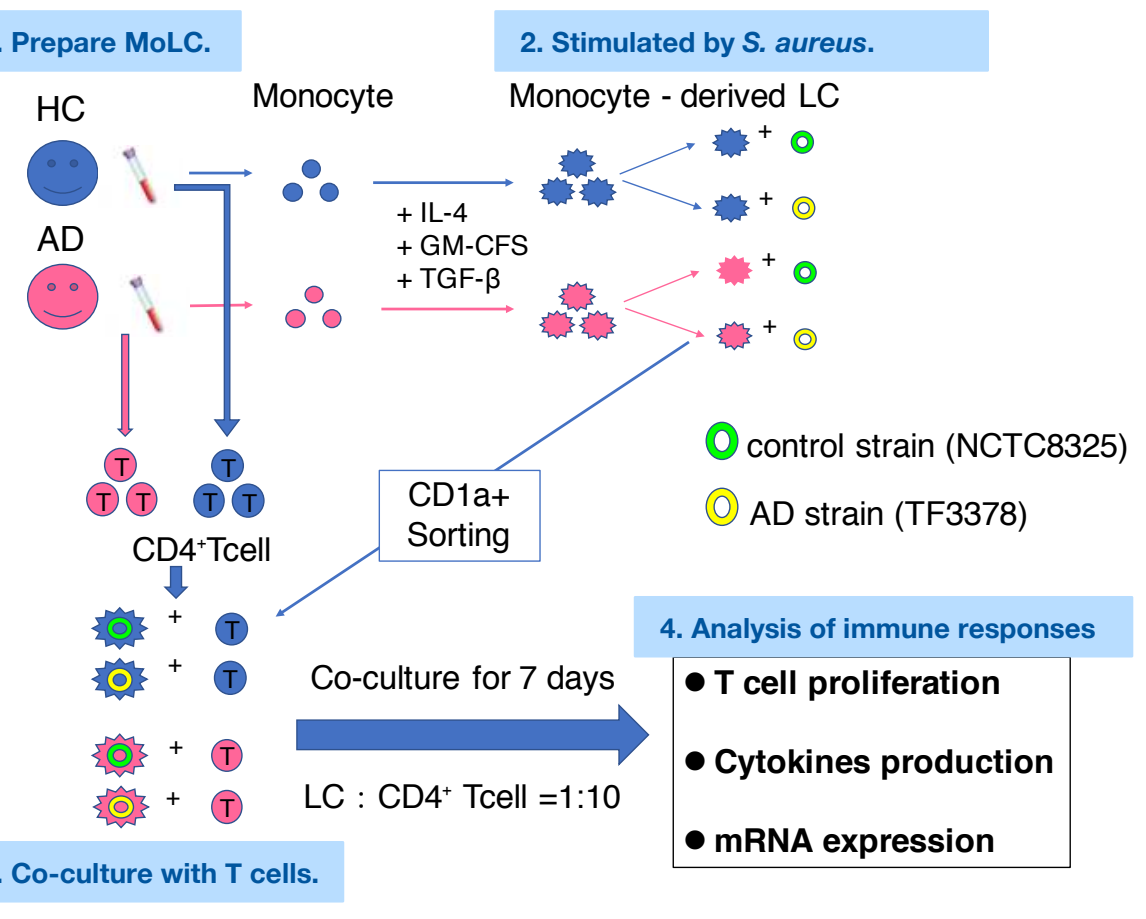

\section{Material \& Method - 2}

The detailed backgrounds of participants and the sequence types of $S$. aureus strains are listed below.

\begin{tabular}{|lcc|} 
& HC $(\mathbf{n}=\mathbf{2 4})$ & AD $(\mathbf{n}=\mathbf{1 3})$ \\
\hline Male : Female & $14: 10$ & $10: 3$ \\
\hline Age & $29 \pm 2$ & $30 \pm 2$ \\
\hline $\operatorname{lgE}(\mathrm{IU} / \mathrm{ml})$ & $133 \pm 59$ & $13667 \pm 5105$ \\
\hline TARC $(\mathrm{pg} / \mathrm{ml})$ & $265 \pm 24$ & $3744 \pm 1240$ \\
\hline
\end{tabular}

\begin{tabular}{|lc|}
\hline S. aureus strain & Sequence type \\
\hline NCTC8325 & 8 \\
\hline TF3378* & 59 \\
\hline TF2962* & 59 \\
\hline TF2868* & 121 \\
\hline TF2870* & 20 \\
\hline
\end{tabular}

Statistical analyses; Paired samples were evaluated using the Repeated Measures ANOVA followed by the NewmanKeuls Multiple Comparison Test or the Wilcoxon matched-pairs signed rank test. For independent samples, the MannWhitney $U$ test was used. Graphs were prepared as scatter plots with mean \pm SEM. Significance was indicated compared to unstimulated control (Ø) and between groups by ${ }^{*} \mathrm{p}<0.05,{ }^{* \star} \mathrm{p}<0.01$ and ${ }^{\star \star \star} \mathrm{p}<0.001$.

Result 1 : S. aureus induces maturation of MoLC.

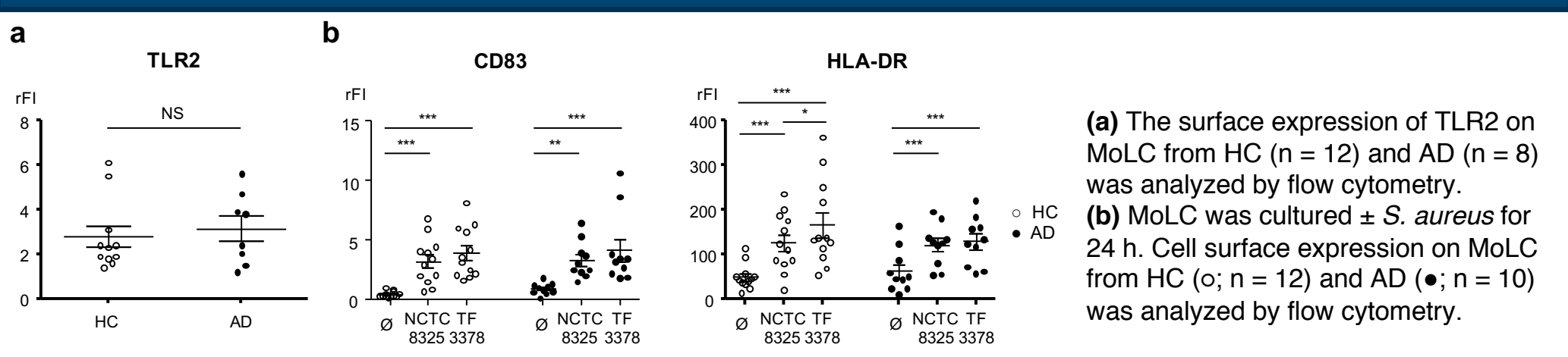

Result 2 : S. aureus from AD skin enhance T cell proliferation.

a

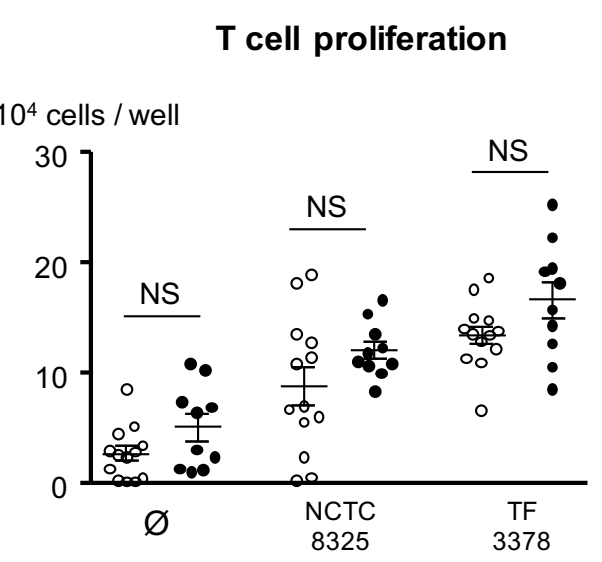

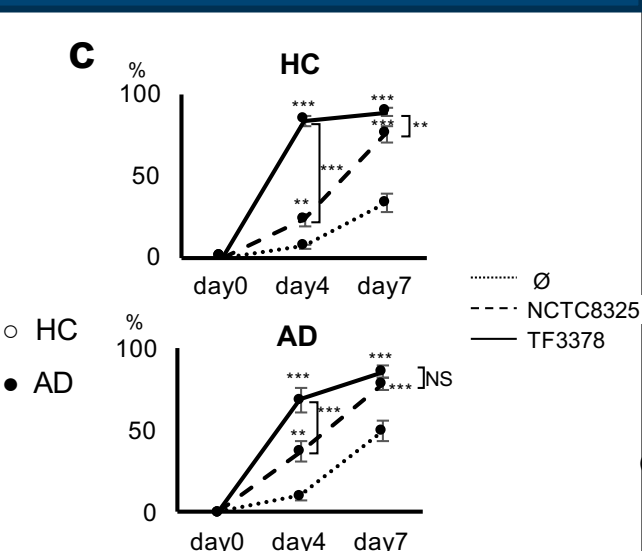

d

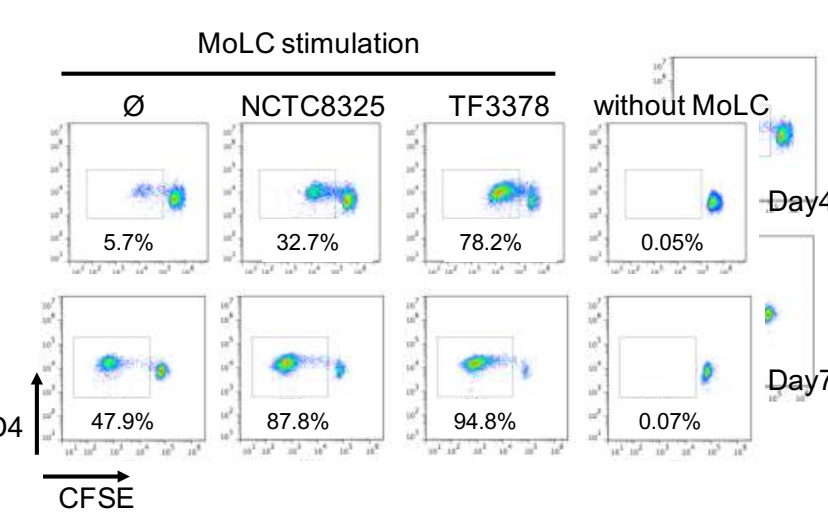

MoLC was cultured $\pm S$. aureus for $24 \mathrm{~h}$. After $S$. aureus stimulation, CD1a-sorted MoLC were co-cultured with autologous CD4pos T cells for 7 days. After co-culture, accurate numbers of pro
cells (7-AADeg) were measured by FACSAriatm (a) MoLC was stimulated by a standard strain of $S$, aureus NCTC8325 or $S$. aureus strains (TF3378, TF2962, TF2868 and TF2820) from AD skin and the number of proliferated T cells was counted, $n=6(\mathrm{HC})$. (b) T cell proliferation by $S$. aureus-stimulated MoLC derived from HC and $A D$ were compared, $\mathrm{n}=13(\mathrm{o} ; \mathrm{HC})$ and $\mathrm{n}=10(\bullet ; \mathrm{AD})$.

(c) The time course of divided T cells was assessed by CFSE, $n=9-13$ (HC) and $\mathrm{n}=6-10$ (AD) (d) A representative dot blot is shown. The percent of divided T cells is

Result 3 : S. aureus TF3378 from AD skin stimulated-MoLC trigger altered cytokine production from $\mathrm{T}$ cells.

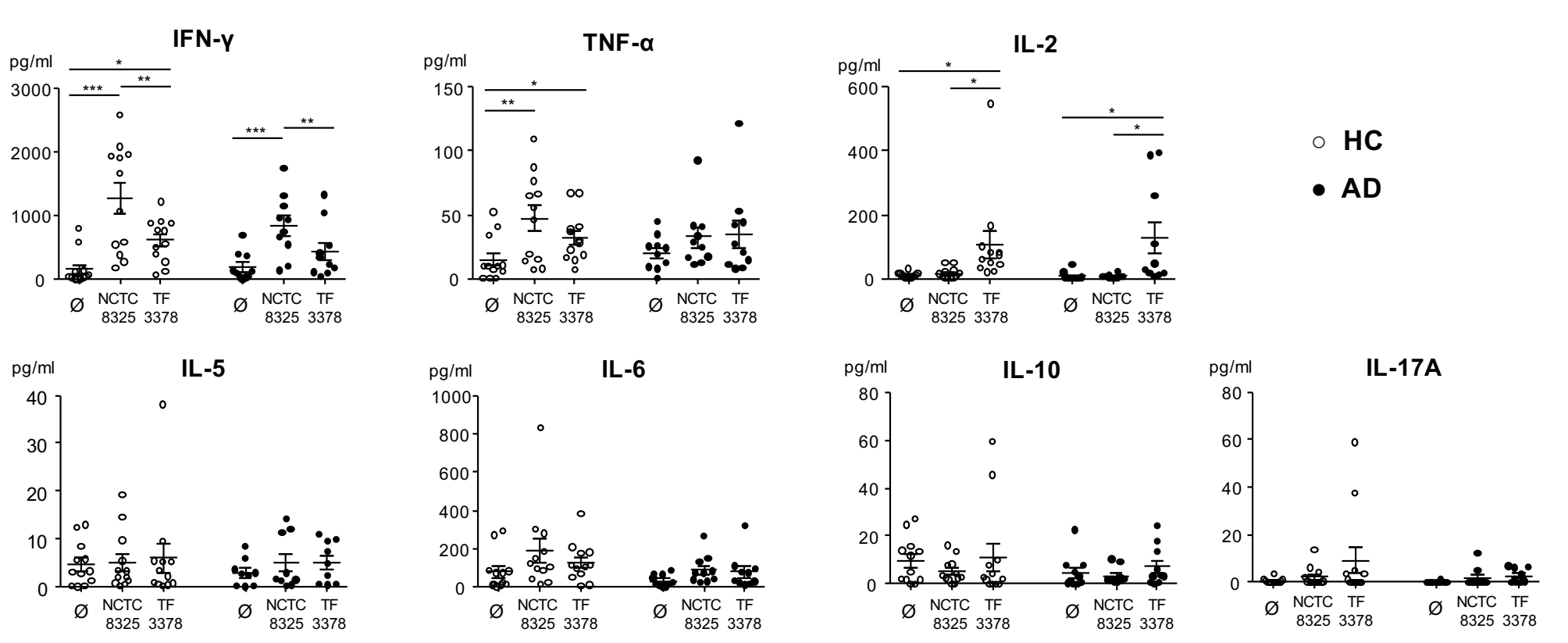

Cytokine production in the supernatant from co-culture was quantified by a BDTM Cytometric Bead Array kit, $n=13(\circ ; H C)$ and $n=9-10(\bullet ; A D)$. The $Y$-axis represents cytokine production per 50,000 live CD4pos T cells.

Result 4 : Quantitative real-time PCR analysis of cytokine mRNAs.

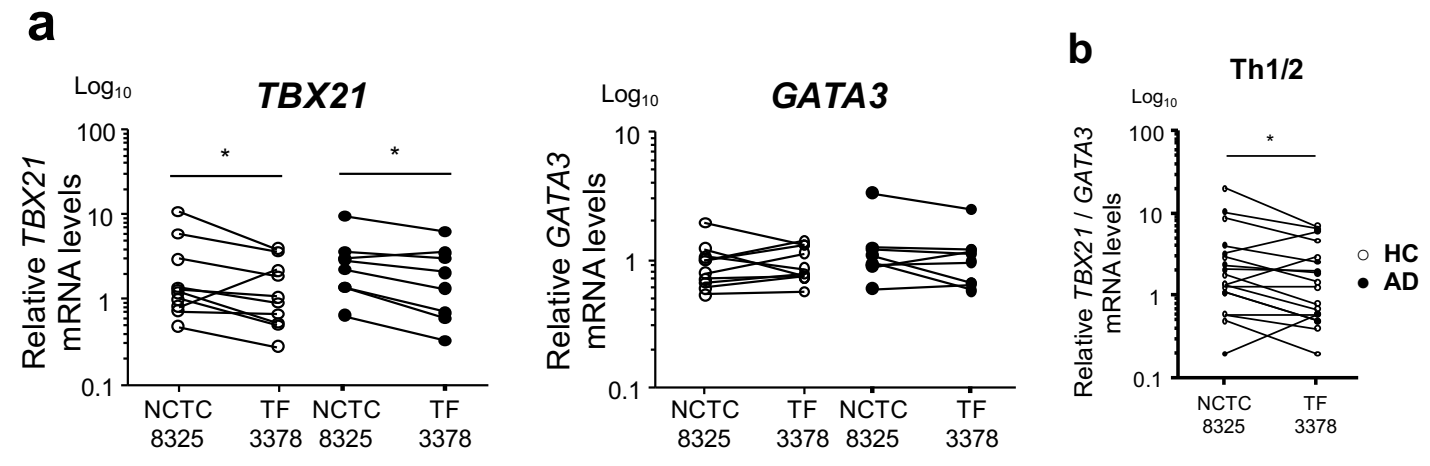

After co-culture, alive CD4pos T cells (7-AAD neg) were collected by FACSAria' II and mRNA was extracted. (a) The expression of

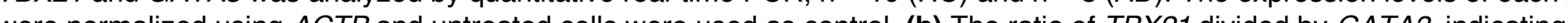
Th1/Th2 balance, was shown.

Result 5 : S. epidermidis induce T cell proliferation via MoLC comparable to a control strain of $S$. aureus

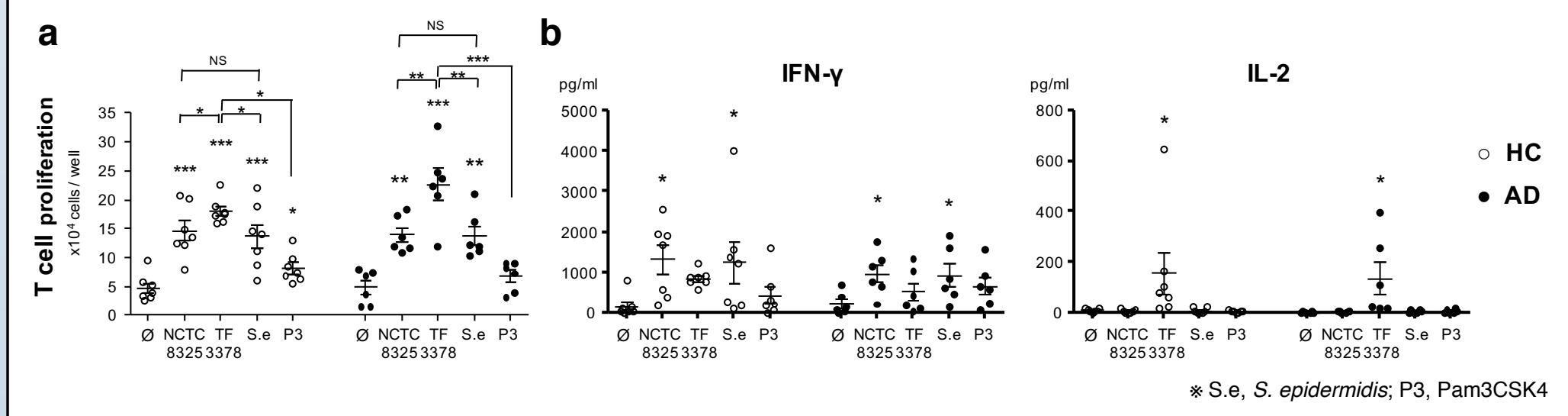

MoLC was cultured \pm S. aureus (NCTC8325 or TF3378), S. epidermidis, or TLR1/2 ligand Pam3CSK4 for $24 \mathrm{~h}$.

(a) Stimulated MoLC was sorted by CD1a and then co-cultured with autologous $C D 4$ pos $T$ cells for 7 days. After co-culture, the number of proliferated alive CD4pos $T$ cells (7-AAD neg) were counted by FACSAriaTM $I I, n=7(0 ; H C)$ and $n=6(\bullet ; A D)$. (b) Cytokine production in the supernatant from co-culture was quantified by a BDTM Cytometric Bead Array kit, $\mathrm{n}=7(\mathrm{o} ; \mathrm{HC})$

Result 6 : Proteinase K treatment of $S$. aureus TF3378 diminish the capacity for activating MoLC.

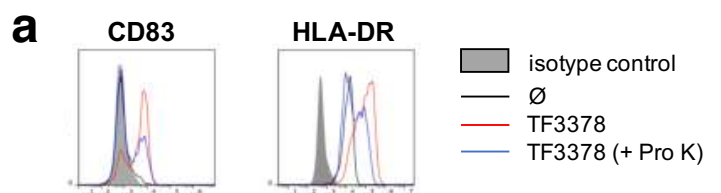

b
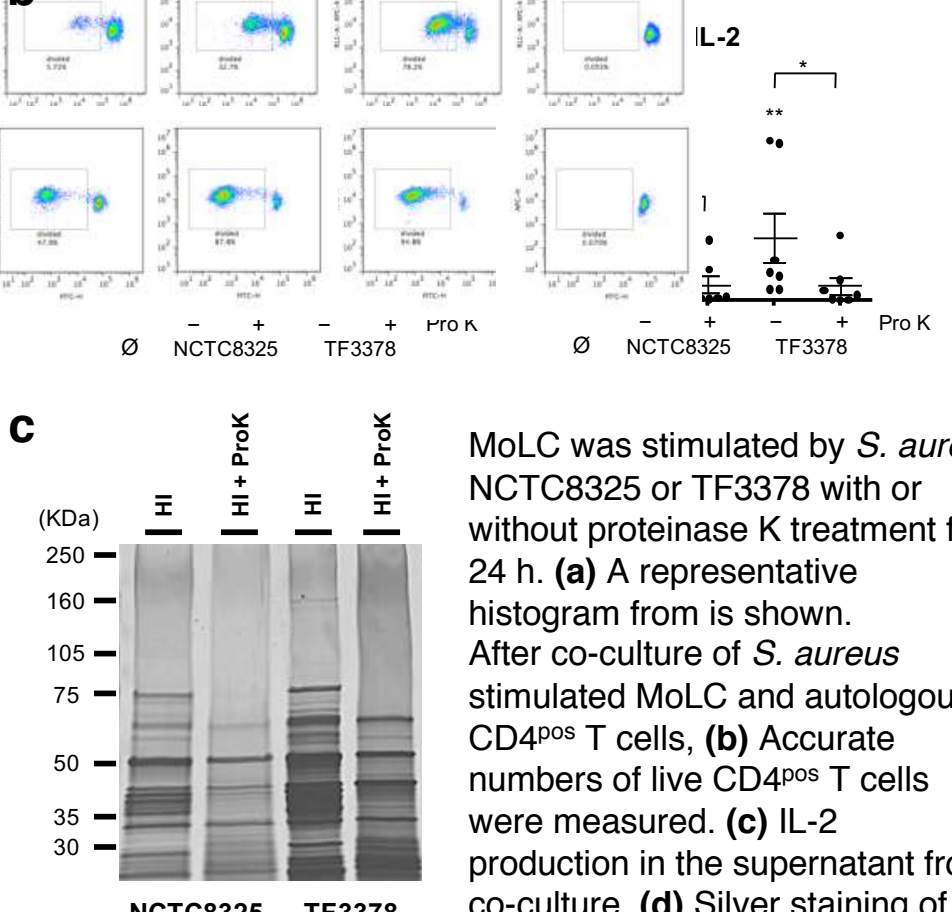

MoLC was stimulated by S. aureus NCTC8325 or TF3378 with or without proteinase $K$ treatmen 24 h. (a) A representative histogram from is shown. stimulated MoLC and autologous CD4pos T cells, (b) Accurate numbers of live (bD4pos $T$ cells were measured. (c) IL-2 production in the supernatant from co-culture. (d) Silver staining of
cell wall proteins extracted from aureus is shown
Conclusion

TLR2 stimulus due to low expression TLR2.

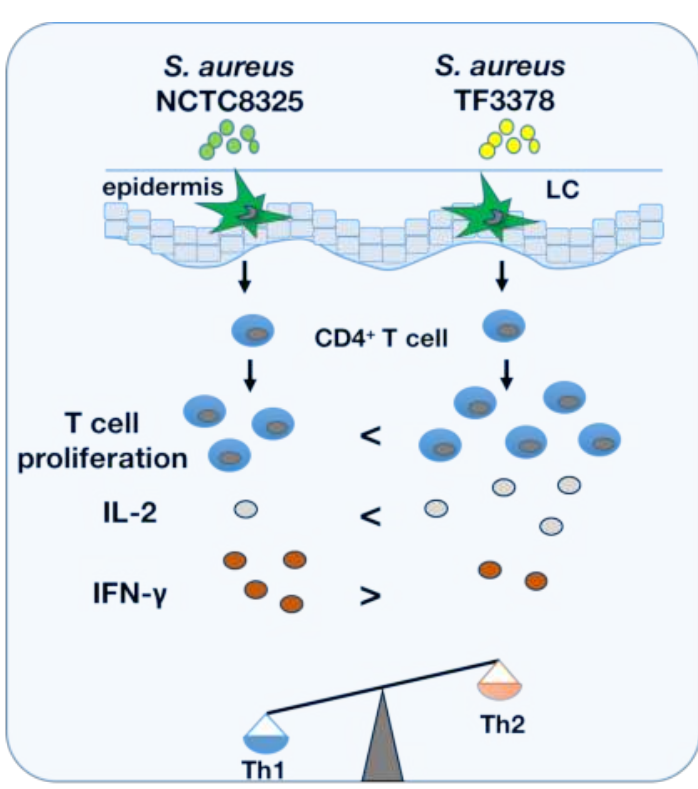

- $A D$ and control strains of $S$. aureus induce different T cell responses via LC

- S. aureus TF3378, AD strain, triggers increased IL-2 and less IFN-y production.

- Altered T cell responses could be due to differences in cell wall proteins of $S$. aureus. Acknowlegment 